\title{
EARLY POSTOPERATIVE MASTICATORY EFFICIENCY AFTER SURGICAL EXTRACTION OF A PARTIALLY IMPACTED MANDIBULAR THIRD MOLAR
}

\author{
D. Konstantinova ${ }^{1}$, A. Nenova-Nogalcheva ${ }^{2}$, P. Pechalova ${ }^{3}$ \\ ${ }^{1}$ Department of Prosthetic Dentistry, Faculty of Dental Medicine, Medical University - Varna, Bulgaria \\ ${ }^{2}$ Department of Oral and Maxillofacial Surgery, Faculty of Dental Medicine, Medical University - Varna, Bulgaria \\ ${ }^{3}$ Department of Oral Surgery, Faculty of Dental Medicine, Medical University - Plovdiv, Bulgaria
}

\begin{abstract}
The extraction of mandibular third molars is a traumatic procedure accompanied by marked clinical symptoms in the postoperative period. The aim of the present study was to determine the effects of the removal of a partially impacted mandibular third molar on the masticatory efficiency in patients in the early postoperative period. Material and methods. A total of 47 participants, divided into two groups, took part in this pilot study. The first group included 33 patients with a mean age of 18.3 years ( $S D=0.9$ years), defined as the Control group; the second group involved patients with a mean age of 33.3 years $(S D=1.86)$, referred to as the Clinical group. The masticatory efficiency of the Clinical group patients was objectively double-checked using a Protab ${ }^{\circledR}$ test bite: once during the preliminary examination (prior to extraction of the wisdom tooth) and again 7 days after the surgical removal of the wisdom tooth. A sieve analysis was applied to determine the artificial test bite particle size and size distribution following mastication. Feedback on the Clinical group patients' assessment of any subjective complaints was obtained through a questionnaire containing two questions considered relevant to patients' masticatory efficiency. The data from the sieve analysis of each fraction were analyzed using the Statistics Software Package for Epidemiological and Clinical Research (IBM SPSS V.20.00). Results. The data processing of all parameters tested prior to and after extraction of the wisdom tooth revealed statistically significant differences. Only the duration of chewing after the third molar extraction matched the chewing duration in the healthy control subjects $(p=0.198)$. The analysis of the feedback on the self-assessment of patients from the Clinical group demonstrated that prior to extraction all subjects (100\%) experienced pain to a varying degree, whereas in half of the patients (50\%) the pain disappeared following extraction. The Spearman's test for correlation between mean particle sizes as well as the questionnaire feedback indicated a strong positive relationship with food trapping. Accordingly, the larger mean particle size was linked to the self-assessment of food trapping around the wisdom tooth prior to extraction (Spearman's Rho $=0.57, p=0.032$ ). There was also a correlation between the chewing time and the sensation of pain in the teeth adjacent to the extraction wound. The results suggested that the shorter duration of chewing was associated with a greater sensation of pain (Spearman's Rho $=-0.61, p=0.026$ ). Conclusion. The eruption of mandibular third molars causes difficulties in the masticatory process. A positive trend was observed towards normalizing of the chewing duration following extraction $(t=-1.356, p=0.198)$.
\end{abstract}

Key words: mandibular third molar, masticatory efficiency, surgical extraction

Corresponding author: Petia Pechalova, Department of Oral Surgery, Faculty of Dental Medicine, Medical University of Plovdiv, 3 Hristo Botev Blvd., Plovdiv 4000, Bulgaria, e-mail: pechalova@abv.bg 


\section{INTRODUCTION}

The retention of third molars is a common pathology in everyday dental practice. There are two types of tooth retention - full retention where the tooth is completely covered with bone, and partial retention where only part of the tooth is covered by bone [1]. The most common treatment method of impacted and partially impacted teeth is odontectomy, i.e. their surgical extraction [2]. The surgical removal of mandibular third molars is a traumatic procedure accompanied by more marked clinical symptoms in the postoperative period [3]. Among the most common postoperative complications are swelling, restricted opening of the mouth and subjective sensation of pain, which affects the masticatory process and impairs the patients' quality of life.

The aim of the present study was to determine whether the removal of a partially impacted mandibular third molar affected the patients' masticatory efficiency in the early postoperative period.

\section{MATERIAL AND METHODS}

A total of 47 participants, divided into two groups, took part in this pilot study. The first group included 33 patients with a mean age of 18.3 years $(S D=$ 0.9 years), defined as the Control group; the second group involved 14 patients with a mean age of 33.3 years $(S D=1.86)$, referred to as the Clinical group. The participants were selected following extra- and intraoral examinations. A general requirement for all participants was to have no concomitant diseases. The inclusion criteria for all participants were as follows: age between 16 and 35 years; lack of active diseases of the hard dental tissues, dental pulp and surrounding soft tissues; full dentition, without extracted teeth; normal function of the temporo-mandibular joints; absence of para-functional activities (bruxism etc.). The difference between both groups was the grade of eruption of the third mandibular molar: the persons with normally erupted mandibular third molars were included in the control group, while patients with partially erupted third mandibular molars were referred to a clinical group.

Surgical procedures were performed in the clinical group under inferior alveolar block anesthesia with Ubistesin forte TM (3M Deutschland $\mathrm{GmbH}$, Germany). The removal of partially impacted mandibular third molar started with a triangular mucoperiosteal incision - a horizontal incision around the distal aspect of the tooth and a releasing incision, located vestibularly; elevation of the muco-periosteal flap; re- moval of the surrounding bone or dividing the tooth in two parts; removal of the tooth; cleaning the socket with saline; covering the socket with the flap, and suturing.

All patients were informed about the nature of the study and its compliance with the UN Universal Declaration of Human Rights, the World Medical Association (WMA) Declaration of Helsinki on ethical principles for medical research involving human subjects, the Convention for the protection of Human Rights and Dignity of the Human Being (Oviedo, 1997) as well as the Health Act of the Republic of Bulgaria. All participants signed an Informed Consent.

The masticatory efficiency of the Clinical group patients was objectively double-checked using a Pro$\operatorname{tab} \otimes$ test bite [4] - once during the preliminary examination (prior to extraction of the wisdom tooth) and again 7 days after the surgical removal of the wisdom tooth. The control subjects underwent only one examination. A sieve analysis was applied to determine the artificial test bite particle size and size distribution following mastication. The study relied on a set of sieves (Retsch, Germany) with aperture sizes of $200 \mu \mathrm{m}, 315 \mu \mathrm{m}, 500 \mu \mathrm{m}, 630 \mu \mathrm{m}, 1.00 \mathrm{~mm}$ and $2.00 \mathrm{~mm}$, arranged in a descending order of sieve sizes on a vibrating sieving equipment (Kern, Germany). The test material obtained after chewing was placed on the top sieve with the largest aperture size and the bolus was rinsed in $1200 \mathrm{ml}$ of water. The collector at the bottom of the sieves gathered the washed liquid and the amount of test food with a particle size of less than $200 \mu \mathrm{m}$. The whole set of sieves was then stored in a drying cabinet at 60 $\pm 2^{\circ} \mathrm{C}$ for 20 minutes for desiccation of the washed particles. The test sieves were then placed onto the sieve shaker, where the oscillation amplitude was 1 $\mathrm{mm}$, the cycle was 10 seconds and the duration was 2 minutes.

Another $60^{\circ} \mathrm{C}$ desiccation session was carried out in a drying cabinet for 4 hours to achieve the required residual moisture. The amount of particles retained on each sieve surface was determined using an electronic scale (0.01g Accuracy). Feedback on the Clinical group patients' assessment of any subjective complaints was obtained through a questionnaire containing two questions considered relevant to the patients' masticatory efficiency.

Question 1: Is there any food trapped in the area of the removed wisdom tooth? The answering options included:
a) No, there is no food trapped.
b) Yes, but the after mouth rinsing the trapped food is removed. 
c) Yes, there is food trapped which is very difficult to remove.

d) Yes, there is food trapped which cannot be removed by me.

Question 2: Do you feel any pain in the wound area of the removed wisdom tooth when teeth are clenched or during chewing? The answering options included:

a) No, never.

b) Yes, only sometimes when teeth are clenched and/ or when eating hard food.

c) Yes, when chewing certain foods.

d) Yes, even when the teeth are relaxed.

The data from the sieve analysis of each fraction were analyzed using the Statistics Software Package for Epidemiological and Clinical Research (IBM SPSS V.20.00).

The statistical methods included: descriptive statistics for quantitative and qualitative data, parametric (Pearson) and non-parametric (Spearman) correlation tests, parametric (T-test) and nonparametric (Mann-Whitney $U$ Test) tests for independent group comparison, parametric (T-test) and nonparametric (Wilcoxon Signed-Ranks Test) tests for related samples, methods of graphical imaging.

\section{RESULTS}

The chewing cycles, taking place in the oral cavity from the initial placing the bite in the mouth to its fragmentation and then passing toward the digestive tract, are marked by the intrinsic characteristics of each subject: a total number of chewing movements (CMs), chewing movements within a second, chewing time (CT) measured in seconds, fragmented particles size after mastication. The results from the study of these parameters for both patient groups' are presented in Table 1.

The data analysis indicated that there were significant differences across all parameters under study prior to and following extraction. Only the chewing duration after extraction of the wisdom tooth proved being close to the chewing duration in the Control group patients $(p=0.198)$. It is likely that the surgical removal of the third molar produces a nervous muscular reaction that leads to a masticatory process similar in duration to that of healthy subjects.

The results displayed in Table 1 demonstrated the differences in the masticatory process not only during the postoperative period but also during the eruption of the mandibular third molar when subjective symptoms often force patients to visit a dental practitioner.

The analysis of the feedback on the self-assessment of patients from the Clinical group revealed that prior to extraction all subjects $(100 \%)$ experienced pain to a varying degree, whereas in half of the patients $(50 \%)$ the pain disappeared following extraction. When teeth were tightly clenched or when eating very hard food before extraction $28.60 \%$ of patients reported pain, as opposed to only $14.30 \%$ following extraction The most negative response ("Yes, I have pain even when the teeth are relaxed.") was selected by more than $1 / 3$ of patients $(35.70 \%)$ prior to extraction and only by $7.10 \%$ after extraction. It can

Table 1. Mean values, standard deviations and statistical differences between the number of chewing movements, chewing duration, number of chewing movements within a second and the mean particle size after chewing

\begin{tabular}{|c|c|c|c|c|}
\hline Parameters & $\begin{array}{l}\text { M (SD) } \\
\text { Clinical group }\end{array}$ & $\begin{array}{l}\text { M (SD) } \\
\text { Control group }\end{array}$ & $T$ & $\mathrm{p}$ \\
\hline Number of CMs (prior to extraction) & $32.36(7.85)$ & \multirow{2}{*}{$49.91(3.28)$} & -8.366 & 0.000 \\
\hline Number of CMs (following extraction) & $30.21(6.75)$ & & -10.917 & 0.000 \\
\hline CT in seconds (prior to extraction) & $39.50(5.84)$ & \multirow{2}{*}{$44.24(3.38)$} & -3.036 & 0.010 \\
\hline CT in seconds (following extraction) & $42.57(4.60)$ & & -1.356 & 0.198 \\
\hline Number of CMs in seconds (prior to extraction) & $0.82(0.15)$ & \multirow{2}{*}{$1.13(0.87)$} & -7.841 & 0.000 \\
\hline Number of CMs in seconds (following extraction) & $0.71(0.12)$ & & -13.051 & 0.000 \\
\hline Mean particle size (prior to extraction) & $1.86(0.53)$ & \multirow{2}{*}{$1.16(0.05)$} & 4.879 & 0.000 \\
\hline Mean particle size (following extraction) & $2.05(0.47)$ & & 7.162 & 0.000 \\
\hline
\end{tabular}


be safely concluded that the difficult eruption of the mandible third molar leads to pain during occlusion in patients from the Clinical group which in turn causes differences in the masticatory process compared to patients from the Control group.

The statistical processing of the results from Question 1 (Is there any food trapped in the area of the removed wisdom tooth?) indicated that the respondents chose mainly between answer a) and answer b). Accordingly, prior to extraction, the response selected was a) No, there is no food trapped $(71.40 \%)$, and after extraction the answer chosen was b) Yes, but the after mouth rinsing the trapped food is removed (71.40\%). The most negative answer d) was not identified by any respondent before or after the wisdom tooth extraction.

The Spearman's test for correlations between mean particle sizes and the questionnaire feedback indicated a strong positive relationship with food trapping. Accordingly, the larger mean particle size was linked to the self-assessment of food trapping around the wisdom tooth prior to extraction $(r S=0.57, p=$ 0.032). There was also a dependence of chewing time on the sensation of pain in the teeth adjacent to the extraction wound. The results suggested that the shorter duration of chewing was associated with a greater sensation of pain $(r S=-0.61, p=0.026)$.

\section{DISCUSSION}

The chewing function reflects the physical act of food mastication [5]. The energy released through muscle contraction drives the jaw to make contact between the upper and lower teeth rows. In normal physiology this is called mastication power. As early as 1893 Black [6] found out that the masticatory power depended on the patients' individual characteristics, such as age, gender, type of food, parafunctional habits, dentition status, etc. These findings were corroborated by the present study. The partially impacted third molar affected the chewing function during its eruption and after extraction. Anderson and Lasserre, cited by Chakalov [7], detected differences in the maximum masticatory forces. The present study also registered statistically significant differences in the parameters between patients in the Control group and the Clinical group $(p$ $<0.01$ ). The only exception was seen in the duration of chewing in patients after the wisdom tooth extraction $(p=0.198)$. It is quite similar to that of healthy control subjects. Similar results have also been confirmed by a number of other studies where the degree of food fragmentation was proved to vary considerably across individuals [8, 9, 10, 11, 12, 13]. The number of chewing cycles, responsible for the preparation of different types of food for the act of swallowing, appears to be relatively constant within a group of patients, regardless of their individual differences [14]. Although mastication seems like a simple process, there are many factors involved according to Bornhorst and Singh [15] - namely the physiological characteristics of the individual performing the chewing action, such as facial anatomy, gender, age, time of day, dentition status, as well as properties of the food being chewed, such as hardness, moisture content, fat content, food portion size, and food structure.

\section{CONCLUSIONS}

The eruption of mandibular third molars causes difficulties in the masticatory process. A positive trend was observed towards normalizing of the chewing duration (in seconds) following extraction ( $t=$ -1.356, $p=0.198)$. The subjective study based on feedback from the patients in the Clinical group replicated to a great extent the results of the objective study of their masticatory efficiency. The obtained results suggested that the variations in the masticatory process in patients from the Clinical group compared to patients from the Control group were due to the eruption of the mandibular third molar rather than the extraction wound.

\section{Conflicts of interest}

The authors declare no conflicts of interest.

\section{REFERENCES}

1. Tsvetanov Ts. Tooth retention., Medical review. 2015; 51 (1): 25-30 [in Bulgarian].

2. Tsvetanov Ts, Bakardzhiev A. Tooth retention. Epidemiological, clinical, X-ray and remedial studies. Dental Medicine. 2012; 94(2): 138-144 [in Bulgarian].

3. Pavlov NB. Coronectomy of impacted and semi-impacted mandibular third molars. Dissertation, Medical University Varna, 2017 [in Bulgarian].

4. Konstantinova D. Study of the masticatory function in experimental and clinical conditions. PhD Dissertation , 2016,Varna

5. Pavlov IP. Lectures on the work of the main digestive glands. USSR Academy of Sciences Publishing House, Science Series, 1949 [in Russian]. 
6. Rowlett, AE. The Gnatho-dynamometer and its use in dentistry, Proc R Soc Med. 1933 Feb;26(4):463-71.

7. Chakalov I. Wear resistance of dental materials. In vitro researches, Dissertation, Sofia, 2014 [in Bulgarian].

8. Fontijn-Tekam FA, Van Der Bilt A, Abbink JH, Bosman F. Swallowing threshold and masticatory performance in dentate adults, Physiol Behav, 2004, 83, 431-436.

9. Hoebler C, Karinthi A, Devaux MF et al. Physical and chemical transformations of cereal food during oral digestion in human subjects. Br J Nutr, 1998, 80, 429-36.

10. Jiffry MTM. Analysis of particles produced at the end of mastication in subjects with normal dentition, J Oral Rehab, 1981, 8, 113-119.
11. Jiffry MTM. Variations in the particles produced at the end of mastication in subjects with different types of dentition, J Oral Rehab 1983, 10, 357-362.

12. Lucas PW, Luke DA. Is food particle size a criterion for the initiation of swallowing, J Oral Rehab, 1986, 13, 127-136.

13. Peyron MA, Mishellany A, Woda A. Particle size distribution of food boluses after mastication of six natural foods, J Dent Res, 2004; 83(7): 578-582

14. Woda A, Foster K, Mishellany A, Peyron MA. Adaptation of healthy mastication to factors pertaining to the individual or to the food, Physiol Behav, 2006, 89, 28-35.

15. Bornhorst GM, Singh RP. Bolus formation and disintegration during digestion of food carbohydrates. Comprehensive Reviews in Food Science and Food Safety, 2012, 11, 101-118. 